\title{
Increased Phase Synchronization of Brainwaves Induced by Specific Music
}

\author{
Ming-Chi Lu, ${ }^{1}$ Yan-Lin Zhong, ${ }^{1}$ Chen-An Chan, ${ }^{1}$ Ying-Jung Lin, ${ }^{2}$ \\ $\mathrm{Zu}$-Ren Wu, ${ }^{3}$ Chia-Ju Liu, ${ }^{2}$ and Ming-Chung $\mathrm{Ho}^{1 *}$ \\ ${ }^{1}$ Department of Physics, National Kaohsiung Normal University, \\ No. 62, Shenjhong Rd., Yanchao District, Kaohsiung City 824, Taiwan \\ ${ }^{2}$ Graduate Institute of Science Education \& Environmental Education, National Kaohsiung Normal University, \\ No. 62, Shenjhong Rd., Yanchao District, Kaohsiung City 824, Taiwan \\ ${ }^{3}$ Department of Physics, Hebei University, \\ No. 180, Wusi Dong Rd., Lian Chi District, Baoding City, Hebei Province 071002, China
}

(Received June 1, 2019; accepted January 6, 2021)

Keywords: music, phase synchronization, electroencephalography, brainwaves

It is beyond doubt that music can improve people's emotions. However, the interaction between music and the brain is very complicated. In general terms, music can cause the activation of the limbic system, which is related to emotions. The variations of the rhythm, frequency, intensity, and so on, all affect our emotions, and can even produce appeasement effects. This study focused on the influence of music on brainwaves. Unlike classical music, rock music, jazz, and other types of music previously used to research brainwaves, we used a special music that was composed of continuous natural sounds, which contained periodic sounds of several specific frequencies. In addition, this music made subjects sleepy. We used electroencephalography (EEG) to analyze the phase synchronization of brainwaves recorded by brainwave sensors. The results revealed that the phase synchronization index (PSI) of the right-brain gamma band when listening to the experimental music was higher than that when no music was listened to, which meant that the experimental music did have an effect on the right brain. This finding provides a novel entry point for studying the impact of music on brainwaves in the future, and right-brain activation may become a key application of brainwave sensors.

\section{Introduction}

The correlation between brainwaves and related responses has long been a research topic of interest. Electroencephalography (EEG) studies indicated that the enhancement of the gamma (approximately $40 \mathrm{~Hz}$ ) amplitude is significant under different stimulus conditions. ${ }^{(1)}$ The different areas of the brain must therefore communicate through the coherence of the gamma band. ${ }^{(2)}$ There is a lot of evidence that sensory feature binding is accomplished by the phase synchronization of induced neuronal gamma activity, where feature binding refers to the integration of information scattered in different cortical areas. Studies indicated that the phase coupling of gamma activity is also related to selective attention and feature binding. ${ }^{(3-5)}$ The *Corresponding author: e-mail: t1603@mail.nknu.edu.tw https://doi.org/10.18494/SAM.2021.2489 
selective activation of brain regions at $40 \mathrm{~Hz}$ is more important than that with stimuli of other frequencies. ${ }^{(6)}$

Phase synchronization of induced gamma activity may play an important role enabling associations between neuron assemblies. The importance of $40 \mathrm{~Hz}$ may be related to intrinsic neuropathological mechanisms causing, for example, schizophrenia, epilepsy, and migraines. ${ }^{(7)}$ However, Fell et al. and Fries et al. claimed that more data are required to address the interaction between attention and gamma synchronization. ${ }^{(3,8)}$ The neural circuits and the synchronization in the gamma band are abnormal in people with schizophrenia. ${ }^{(9,10)}$ Epileptic seizures are preceded by a decrease of synchronization. A hypersynchronous state of brain dynamics impairs information processing. ${ }^{(11)}$ From the above, it is clear that synchronization and the gamma band, especially at $40 \mathrm{~Hz}$, are of significance in brainwave research.

In this study, we explored the effect of music containing certain frequencies of the gamma band on brainwaves through phase synchronization analysis. Unlike in previous studies, ${ }^{(12-14)}$ the music we used was composed of natural sounds including those with frequencies of $40 \mathrm{~Hz}$ and its multiples. The phase synchronization index (PSI) was used to analyze EEG signals recorded by brainwave sensors. Studies have shown that the right brain is dominant in infants. ${ }^{(15)}$ However, with increasing age, the left brain gradually becomes dominant. So far, no effective method to enhance the right-brain activation has been proposed. Exploiting the right brain is an attractive and promising research topic, although there have been few studies on this area. ${ }^{(15-17)}$ Our experimental result showed a good effect on right-brain activation, which may become an effective application of brainwave sensors.

\section{Materials and Methods}

\subsection{Materials}

Fifteen healthy right-handed young subjects (10 males and 5 females) participated in the study. All were required to give written informed consent. This study conformed to the Declaration of Helsinki and was approved by the ethical committee of National Kaohsiung Normal University. EEG signals were collected from electrodes at 36 locations in accordance with the extended international 10-20 system. The impedance of the electrodes was $5 \mathrm{k} \Omega$. The frequency used to sample the brainwave data was $1000 \mathrm{~Hz}$. The experimental process involved the following three stages. Stage (I): rest for 3 min with the eyes closed. Stage (II): listen to the music, whose frequency as a function of time is shown in Fig. 1, for $12 \mathrm{~min}$. Stage (III): rest for 3 min with the eyes closed. The lights in the experiment room were all turned off throughout the experiment.

The experimental data were filtered in the range of $30-60 \mathrm{~Hz}$ with a 20th-order zero-phase filter, which is conducive to analyzing the phase synchrony of the gamma band. The gamma brainwaves measured from the left-brain electrodes (F7, F3, FT7, FC3, T3, C3, TP7, CP3, T5, and P3) and right-brain electrodes (F4, F8, FC4, FT8, C4, T4, Cp4, TP8, P4, and T6) were used to calculate the PSI and the short-time Fourier transform. 


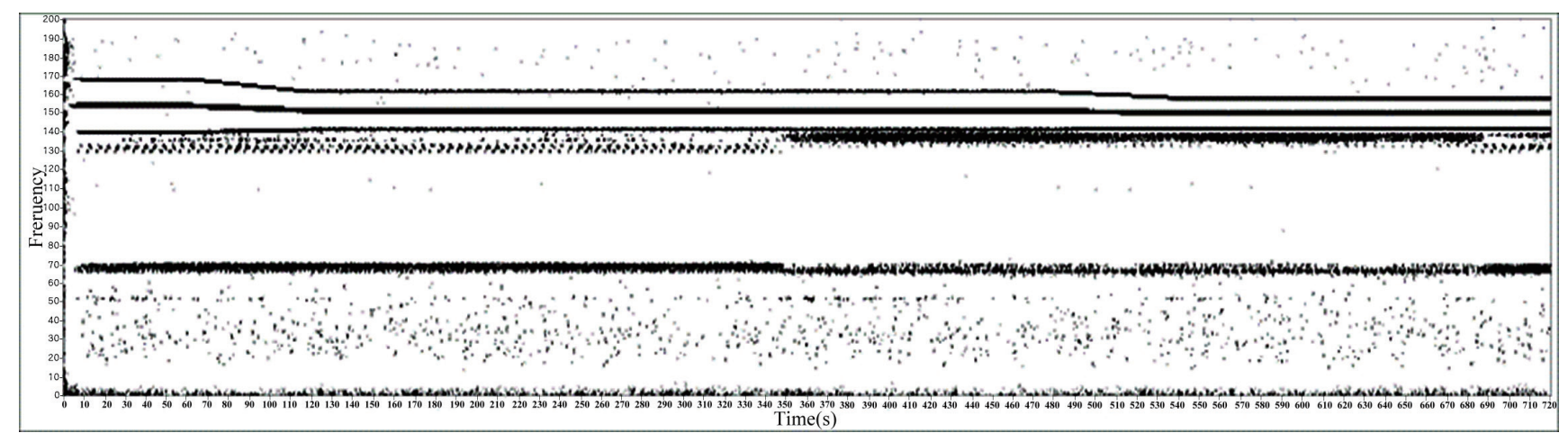

Fig. 1. Time-frequency of the experimental music.

The experimental music was composed of continuous natural sounds such as wind and water, and included periodic sounds with several specific frequencies, as shown in Fig. 1. In addition, this music was interspersed with many low-frequency sounds, called brown noise, which had the effect of soothing the mood and regulating the brainwaves. Many subjects claimed that they could not help falling asleep while listening to the experimental music.

\subsection{Methods}

\subsubsection{Short-time Fourier transform}

To perform the phase analysis, the EEG records, which are time series of amplitude, must first be converted into time series of frequency by a short-time Fourier transform. The timefrequency $s(t)$ of the signal was determined using the short-time Fourier transform, which has the following form:

$$
\operatorname{STFT}(t, f)=\int_{-\infty}^{\infty} w(t-\tau) s(\tau) e^{-j 2 \pi f \tau} d \tau
$$

where $w(t)$ is the window function. We chose a Hann window with a length of 256 sample points and a time shift of $0.01 \mathrm{~s}$.

Then, the time series of the phase was determined using the Hilbert transform, which has the following form:

$$
H(s(t))=\frac{1}{\pi} p \cdot v \cdot \int_{-\infty}^{\infty} \frac{s(\tau)}{t-\tau} d \tau,
$$

yielding a complex analytic signal $S(t)$ formulated as 


$$
S(t)=s(t)+i H(s(t))=A(t) e^{i(w t+\phi)} .
$$

Here, p.v. is the Cauchy principal value, $A(t)$ is the instantaneous amplitude, and $\phi(t)$ is the instantaneous phase.

\subsubsection{PSI}

Consider two coupled neural oscillators $(a, b)$ with phases $\phi_{a}(t)$ and $\phi_{b}(t)$, respectively, whose phase difference is defined as

$$
\phi_{n, m}(t)=n \phi_{a}(t)-m \phi_{b}(t)
$$

where $n$ and $m$ are ratios, both of which are set to 1 here.

The Shannon entropy is defined as

$$
S=-\sum_{k=1}^{N} p(k) \ln p(k) .
$$

Then $S_{\max }=\ln (N)$, where $p(k)$ in Eq. (5) is the phase probability of each bin and $N$ is the number of bins.

The PSI is defined as

$$
\tilde{\rho}_{n m}=\frac{S_{\max }-S}{S_{\max }}
$$

where $0 \leq \tilde{\rho}_{n m} \leq 1 ; \quad \tilde{\rho}_{n m}=0$ indicates no synchronization and $\tilde{\rho}_{n m}=1$ indicates perfect synchronization. Since the EEG data are nonstationary and nonlinear, we performed a sliding window analysis with a window size $T$ of $1 \mathrm{~s}$ and a time shift $t$ of $0.01 \mathrm{~s}$.

\section{Experimental Results}

Figure 2 shows the changes in the PSI of the left and right brains of one subject in the gamma band during the three stages of the experiment. In stage (I), the subjects closed their eyes and rested without performing any task. In stage (II), the subjects listened to the experimental music. During this stage, the subjects were mentally and physically relaxed and even fell asleep. In stage (III), the music stopped and the subjects rested again.

The experimental results revealed that the original PSI of the right brain was lower than that of the left brain during stage (I). In the first half of stage (II), the PSI of the right brain was still lower, but in the second half of stage (II), it began to increase significantly and even overtook that of the left brain. In stage (III), the PSI of the right brain became lower and returned to the same value as in stage (I). In summary, listening to the experimental music enhanced the rightbrain synchronization. 


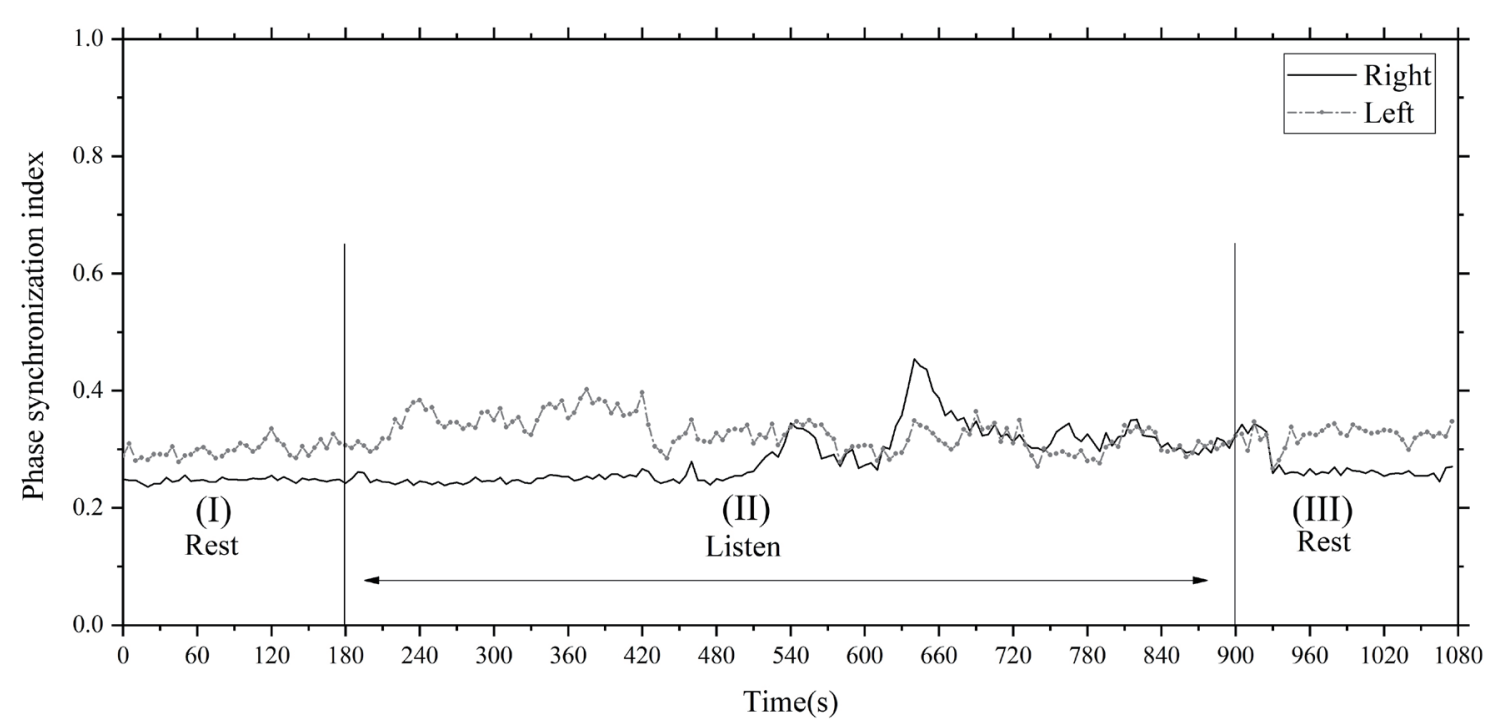

Fig. 2. PSI of left and right brains of one subject in gamma band during the experiment.

\section{Discussion}

Although it was found from the experimental results that listening to the music enhanced the right-brain synchronization, this occurred not at the beginning of listening to the music, but after listening to it for a while (about $6 \mathrm{~min}$ ). When the music stopped playing, the effect of the right-brain synchronization was not immediately weakened, but lasted about $30 \mathrm{~s}$, and then sharply diminished and returned to its original state. In addition, in stage (II), we found that the PSI of the right brain increased, which lasted about $4 \mathrm{~min}$, although that of the left brain did not increase at the beginning of stage (II). In summary, the effect of enhancing the rightbrain synchronization while listening to the music required about 6 min of gestation time, and it lasted about $30 \mathrm{~s}$ when the music stopped playing.

\section{Conclusions}

The enhancement of brain synchronization helps activate the brain. In this study, we proposed an experiment in which music stimulated the right brain, and the PSI of the right brain was effectively increased, which may provide an opportunity to enhance right-brain activation. In addition, this finding provides a novel entry point for studying the impact of music on brainwaves in the future, and right-brain activation may become an effective application of brainwave sensors.

\section{References}

1 O. W. Sakowitz, R. Q. Quiroga, M. Schürmann, and E. Başar: Cognit. Brain Res. 11 (2001) 267. https://doi. org/10.1016/S0926-6410(00)00081-1

2 G. Buzsáki, N. Logothetis, and W. Singer: Neuron 80 (2013) 751. https://doi.org/10.1016/j.neuron.2013.10.002 
3 J. Fell, G. Fernández, P. Klaver, C. E. Elger, and P. Fries: Brain Res. Rev. 42 (2003) 265. https://doi.org/10.1016/ $\underline{\mathrm{S} 0165-0173(03) 00178-4}$

4 R. H. Paul, C. R. Clark, J. Lawrence, E. Goldberg, L. M. Williams, N. Cooper, R. A. Cohen, A. M. Brickman, and E. Gordon: J. Integr. Neurosci. 4 (2005) 63. https://doi.org/10.1142/S0219635205000690

5 S. M. Doesburg, A. B. Roggeveen, K. Kitajo, and L. M. Ward: Cerebral Cortex 18 (2008) 386. https://doi. org $/ 10.1093 /$ cercor/bhm073

6 M. A. Pastor, J. Artieda, J. Arbizu, J. M. Marti-Climent, I. Peñuelas, and J. C. Masdeu: J. Neurosci. 22 (2002) 1051. https://doi.org/10.1523/JNEUROSCI.22-23-10501.2002

7 C. J. Liu, C. F. Huang, C. Y. Chou, M. C. Lu, C. H. Yu, Y. T. Lin, M. T. Wu, and M. C. Ho: Appl. Mech. Mater. 311 (2013) 491. https://doi.org/10.4028/www.scientific.net/AMM.311.491

8 P. Fries, J. H. Reynolds, A. E. Rorie, and R. Desimone: Science 291 (2001) 1560. https://science.sciencemag. org/content/291/5508/1560.full

9 P. J. Uhlhaas and W. Singer: Dialo. Clin. Neurosci. 15 (2013) 301. https://doi.org/10.31887/DCNS.2013.15.3/ puhlhaas

10 Y. W. Shin, B. F. O'Donnell, S. Youn, and J. S. Kwon: Psychiatry Invest. 8 (2011) 288. https://doi.org/10.4306/ pi.2011.8.4.288

11 C. J. Stam: Clin. Neurophysiol. 116 (2005) 2266. https://doi.org/10.1016/j.clinph.2005.06.011

12 T. Särkämö, E. Pihko, S. Laitinen, A. Forsblom, S. Soinila, M. Mikkonen, T. Autti, H. M. Silvennoinen, J. Erkkilä, M. Laine, I. Peretz, M. Hietanen, and M. Tervaniemi: J. Cognit. Neurosci. 22 (2010) 2716. https://doi. org/10.1162/jocn.2009.21376

13 G. M. Bidelman and C. Alain: J. Neurosci. 35 (2015) 1240. https://doi.org/10.1523/JNEUROSCI.3292-14.2015

14 T. Fujioka, B. Ross, and L. J. Trainor: J. Neurosci. 35 (2015) 15187. https://doi.org/10.1523/ JNEUROSCI.2397-15.2015

15 C. Chiron, I. Jambaque, R. Nabbout, R. Lounes, A. Syrota, and O. Dulac: Brain 120 (1997) 1057. https://doi. org/10.1093/brain/120.6.1057

16 U. Dimberg and M. Petterson: Psychophysiology 37 (2000) 693. https://doi.org/10.1111/1469-8986.3750693

17 S. Coulson and Y. C. Wu: J. Cognit. Neurosci. 17 (2005) 494. https://doi.org/10.1162/0898929053279568 